\title{
3D PRINTING OF FIBERS AND REINFORCEMENTS FOR CEMENTITIOUS COMPOSITES TO MAXIMIZE THE FRACTURE-RESISTING PERFORMANCE OF FRCCS
}

\author{
YOUNG JUN NAM* ${ }^{*}$ JI WOON PARK ${ }^{\dagger}$, SEUNG HYUN YOON ${ }^{\dagger \dagger}$ \\ AND YUN MOOK LIM ${ }^{\dagger \dagger \dagger}$ \\ Yonsei University \\ Seoul, Republic of Korea \\ e-mail: raden4@yonsei.ac.kr \\ ${ }^{\dagger}$ Yonsei University \\ Seoul, Republic of Korea \\ e-mail: zertofroot@gmail.com \\ ${ }^{\dagger \dagger}$ Yonsei University \\ Seoul, Republic of Korea \\ e-mail: heritages91@gmail.com \\ ${ }^{\dagger \dagger}$ Yonsei University \\ Seoul, Republic of Korea \\ e-mail: yunmook@yonsei.ac.kr
}

Key words: 3D printing, Fiber Reinforced Concrete, composites, SIFCON, distribution of fibers

\begin{abstract}
To date, FRCCs (Fiber Reinforced Cementitious Composites) have been used with many different applications in various industries. However, the construction field has adopted this system relatively late compared to other industries. As this field shows an increasing need for FRCCs, research in this area has also expanded rapidly. Recently, various cutting-edge technologies have been integrated with the manufacturing of matrices and fibers to develop higher-performance FRCCs. However, the technologies involved in the current stage are usually focused on the development of material properties, the performances of matrix and fibers, and interfaces between matrix and fibers. Among the newly developed technologies, 3D printing has increased in popularity and has been favored over others. Many different applications are currently attempting to utilize 3D printing techniques to enhance performance in an innovative approach to the field of construction. Applications of 3D printing in this field have been developed for new materials and methodologies to print structures directly. In this study, reinforcements, including rebars and fibers, are printed using a 3D printer to make high-performing FRCCs and RC (Reinforced Concrete). In particular, when making FRCCs, fibers are usually mixed with the cement-based matrix during the process. In this method, the distribution of fibers is impossible to control because of the random nature of the fibers' positions. This study focuses on how fibers and reinforcement can be printed in a controlled manner. If the suggested new fiber and reinforcement printing process can be developed, mixing problems such as clumping, poor distribution, and difficulties during mixing will easily be solved with printed fibers and reinforcement using the SIFCON (Slurry Infiltrated Fiber Concrete) process.
\end{abstract}




\section{INTRODUCTION}

Realizing a relationship between steel and concrete in the modern construction industry has long been an important task. While strong in compression, concrete is weak in tension. Concrete is also a quasi-brittle material which means that failure occurs without yielding. To overcome its structural imbalances and to improve its strength and overall ductility, steel reinforcement is added to the crack-initiating parts of concrete. However, even with added reinforcement, $\mathrm{RC}$ has room to improve as a material. Additional reinforcements have to be added to concrete in order to resist abnormal loadings such as seismic or impact loading [1].

As the concept of composite material emerged, FRCCs became popular. From the early 1900s, short lengths of asbestos fibers were mingled with concrete to enhance its tensile behavior. However, once the health risks associated with asbestos were discovered, the need arose to find a replacement for the substance in concrete. Steel, glass, and synthetic fibers such as polypropylene have been used in concrete as replacements. The performance of FRCCs showed differences according to their matrix strength, material type and elastic modulus of fiber, orientation of fibers, the size of aggregates, and other factors $[6,7]$.

Although the benefits of FRCCs have been verified by many researchers, the lack of a technique to distribute the fibers to desired locations creates difficulty in adapting the technique for construction sites. Moreover, even though the directional property of fibers is considered a major factor in the behavior of composites, a methodology to control the orientation of fibers is still lacking [2].

Traditionally, creating an FRCC specimen involved simply mixing fibers into a composite and waiting until the concrete hardened. Using this method, distributing the fiber inside the composite was difficult and caused problems such as fiber balling and maldistribution of fibers.

This research studied improvement of traditional fibers and reinforcements for cementitious composites utilizing a 3D printing technique. Also, structures such as reinforced rebars, aligned reinforcements, and fibers were printed to investigate the possibilities of applying the printer in the civil engineering field. The designed fibers and reinforcements were used to examine the arrangement, distribution, and direction of the fibers. In the case of FRCCs, the randomness of distribution of the fibers is expected to decrease as the fibers were placed in the desired locations.

\section{APPLICATION OF 3D PRINTING IN THE CIVIL ENGINEERING FIELD}

As shown in Figure 1, various types of 3D printing exist and are classified by which material they use. In 3D printing, which is also called "additive manufacturing" or "rapid prototyping", four methods are commonly used: fused deposition modeling (FDM), Stereolithography (SL), Polyjet printing, and selective laser sintering (SLS).

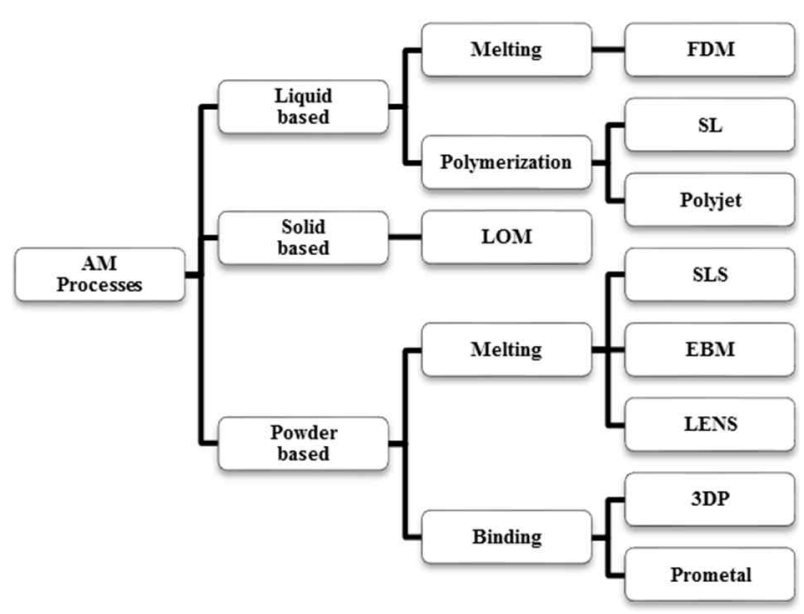

Figure 1: Three-dimensional printing processes [11].

First of all, the FDM printer uses a thermoplastic filament which is heated to its melting point and then extruded layer by layer. The printer repetitively lays down one crosssection of the object after another, as depicted in a design file. Secondly, SL printers have four main parts: a tank that can be filled with liquid plastic, an ultraviolet laser (UV), a perforated platform, and a computer that controls the platform along with the laser. The printer 
sweeps the laser beam over the surface of a UVsensitive photopolymer that hardens when exposed to UV light. Each sweep of the laser traces the outline and cross section of the printed shape in consecutive layers. Thirdly, SLS printers follow a technique similar to SL printers; however, instead of using liquid photopolymers in a vat, SLS printers use powder. SLS printers trace a high-power laser beam over the surface of a bed of powder, fusing the particles into a solid form. Lastly, a Polyjet printer has a carriage with four or more inkjet heads and UV lamps which traverse the workspace, depositing tiny droplets of photopolymers that solidify when exposed to UV light. After printing a thin layer of material, the process repeats until a complete $3 \mathrm{D}$ object is formed. Polyjet printers can use several print heads at once, and so can print multiple materials in a single print job [14].

3Ds printer lacks effectiveness in the civil engineering field compared to their effectiveness in other engineering fields. Utilization of the technology is gradually increasing in other fields such as mechanics, electronics, and medical science. Due to the large scale of infrastructures such as bridges, tunnels, harbors, and dams, along with their unique characteristics depending on the condition of construction sites, civil engineering has been rather slow to apply 3D printing technology. However, application of the technique is steadily increasing.

In civil engineering, research and development are the fields where 3D printing technique performs with relatively greater efficiency. For example, a reduced-sized prototype of a tunnel can be created to examine the effects of seismic and wind loads. Also, use of composite materials has recently increased. Furthermore, a modularized construction method for bridges is being developed and adopted. The use of 3D printing technology for research in the structural analysis might, therefore, accelerate [5].

\section{REINFORCEMENTS THREE DIMENTIONAL MODELING PROCEDURE}

Reinforcement assembly is generally timeconsuming and laborious work. For example, reinforcement stirrups are assembled by spacing them along a main reinforcing steel, after which they are tied to the reinforcing steel. Concrete spacers made of the same material as the poured concrete have to be placed with regard to the cover depth of concrete. The assembling process of mesh reinforcement is complicated, as each reinforcement has to be tied. And as all these processes are done manually, they might experience steel congestion, which interrupts the correct placement and compacting of concrete. Therefore, fabricating reinforcements and adjusting them with a 3D printer will greatly shorten construction processes and solve many of their problems.

In this study, a $220 \times 50 \times 50 \mathrm{~mm}$ specimen was constructed. Considering the size limit for the $3 \mathrm{D}$ printer, three types of reinforcement design were also printed: traditional rebar with stirrups (Figure 2), aligned reinforcements (Figure 3), and randomly distributed fibers (Figure 4). Additional reinforcements were directed towards locations where cracks occur due to a weakness in tensile strength (Figure 3b).

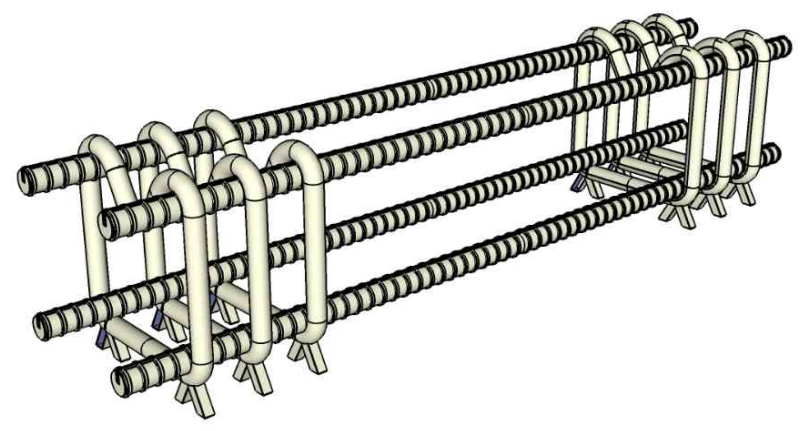

Figure 2: 3D modeling of rebar with stirrups 


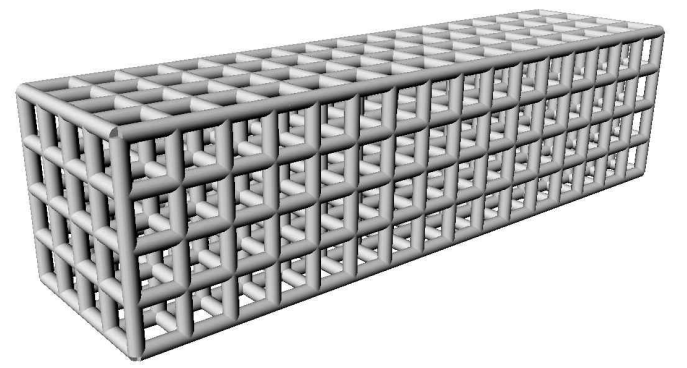

(a) Ordinary mesh form

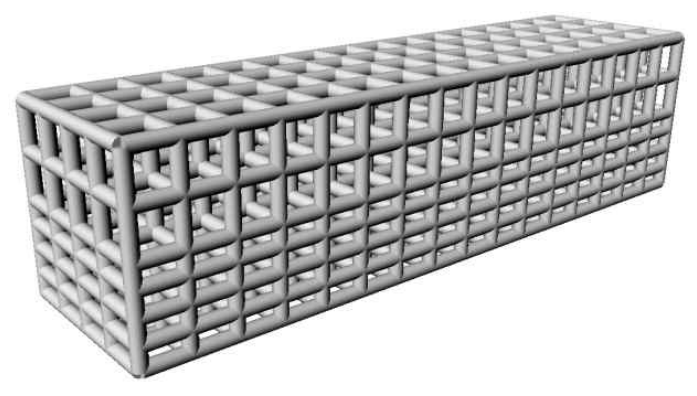

(b) Controlled reinforcement with density

Figure 3: 3D modeling of aligned reinforcement

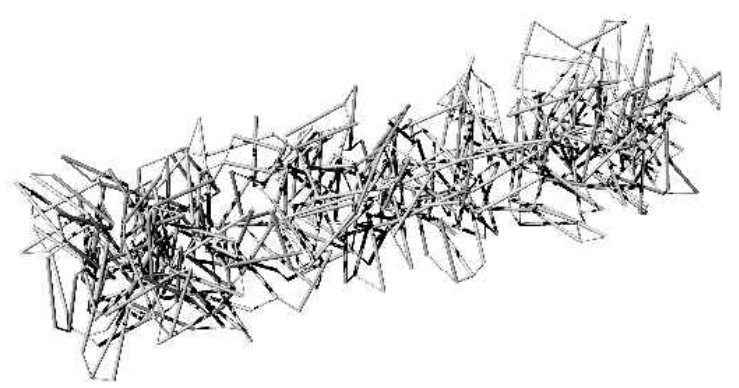

Figure 4: 3D modeling of randomly distributed fibers

Unlike with rebar and aligned reinforcement, the fibers were sufficiently thin and complicated that a 3D printer with high resolution was required. Some difficulties were faced when modeling and printing this structure. Therefore, this section will mainly present the methodology of making the fiber structure.

\subsection{Generating fiber coordinates}

Two kinds of the method were considered when generating points. Firstly, a boundary condition-setting process was conducted to prevent the possibility of some fibers sticking out of the surface of the specimen. As shown in Figure 5, the boundary was created inside the concrete mold, with a distance between the boundary and the mold of one fiber length. As each fiber is composed of two coordinates, the first point was generated inside the boundary using a random function (Figure 5). The second point was developed according to specified variables of length and number of fibers, and random variables of azimuthal angle $(\theta)$ and polar angle $(\varphi)$ (Figure 6). By providing constraints on directional angles $(\theta, \varphi)$, it was also possible to orient the fibers in a more parallel direction to the bottom surface, which will increase the strength of the reinforcement. However, because the first point was placed inside the boundary, it was difficult using the first method to place the fibers close to the point where tensile strength is at its weakest (center bottom of the beam).

To supplement the first method, the second method attempted to distribute the fibers without using a boundary condition. As shown in Figure 7, after generating the first point, if a fiber had a possibility of protruding from the surface, the second point was generated within the angle, $\varphi$. The fibers created from the second method, compared to the first method, were closely located to each other and to the surface of the concrete mold. Therefore, the 3D modeling of randomly distributed fibers used the second method (Figure 4).

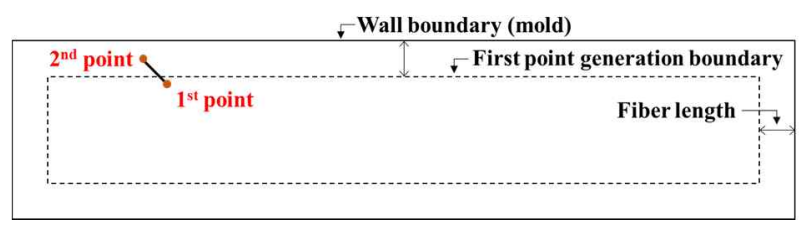

Figure 5: Point generation given the boundary condition

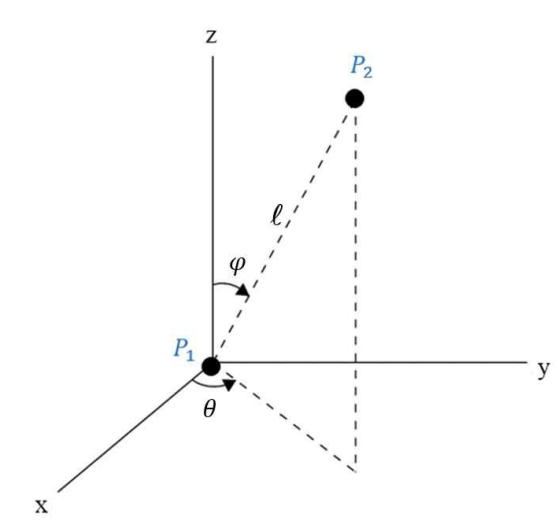

Figure 6: Spherical coordinates $(\ell, \boldsymbol{\theta}, \boldsymbol{\varphi})$ : fiber length $\ell$, azimuthal angle $\boldsymbol{\theta}$ and polar angle $\boldsymbol{\varphi}$ 


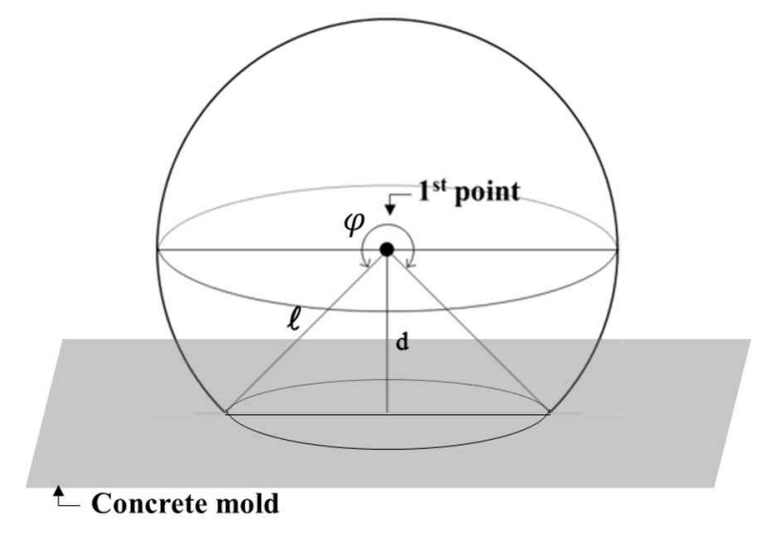

Figure 7: point generation considering the distance between the first point and the concrete mold face.

\subsection{Adjustment for self-sustaining support}

The fibers cannot sustain their formation and structure through distribution alone. To hold the fibers in the desired locations, some kind of support was necessary. Acting as supports, connections between fibers were employed to make the fibers self-sustaining. Given the different behavior when two fibers are joined, connections half the size of the fibers were used.

To connect all the fibers, distances between the fibers were measured and two fibers with the shortest distance between them were joined. Consequently, the fiber structure was built using a three-dimensional modeling program. From Figure 4, some parts of the fibers were extracted for better visualization of the connected fibers (Figure 8 and 9).

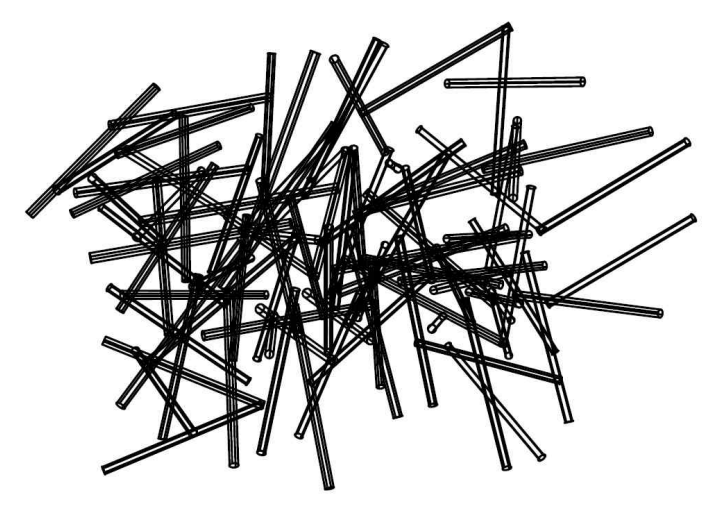

Figure 8: Result of fiber distribution using program

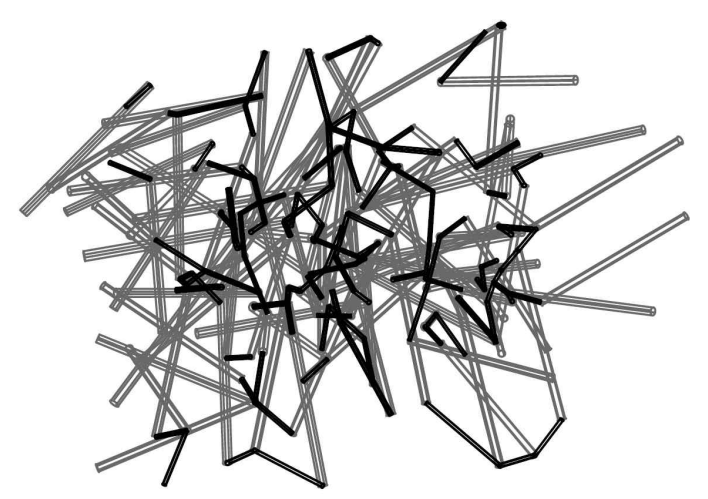

Figure 9: Completed fiber structure by connecting fibers

\section{RESULTS}

First, a model was designed in a 3D modeling program (Rhinoceros 5), which was then translated into an STL (Standard Tessellation Language) file containing information on each layer. Then, the 3D printer manufactured the layers from bottom to top. As 3D printing follows a bottom-up procedure, additional supporting structures had to be printed along with the model, to prevent collapse if overhanging geometries were present. Figure 10 shows the support and frame of the printed structures. Thus, one major step in the 3D fabrication was the placement and removal of supporting structures in printed objects [12].

An FDM printer was used to print three different models. Because a rebar-with-stirrups model and an aligned-fiber model both have thick frames, it was possible to remove the supports for the 3D printing by hand. However, randomly distributed fibers could not be printed using an FDM, due to the low resolution of the printer used. In order to print these fibers, maintaining the original structure without the supporting structures of 3D printing, a printer with high resolution and thin layers was needed. A PolyJet printer was eligible for this task, and the fibers were successfully printed (Figure 11). As 3D printing techniques are rapidly developing, in the near future, more complicated structures might easily be printed.

The following Figures 11, 12, and 13 show printed structures put into a mold with an inner size of $220 \times 50 \times 50 \mathrm{~mm}$. The composite is normally casted. As shown in Figure 12 and 13, 
the mortar was poured into half of the mold to show how it looks inside the cast.

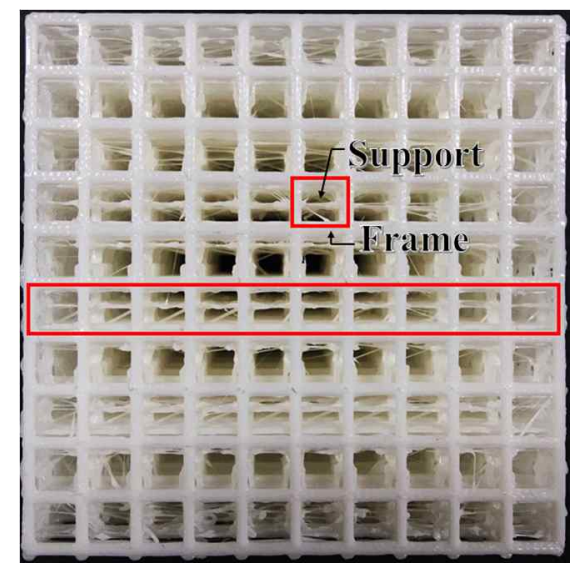

Figure 10: Support and frame created with 3D printing

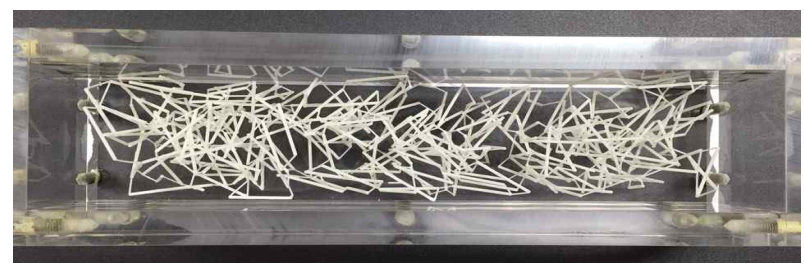

(a) Top view

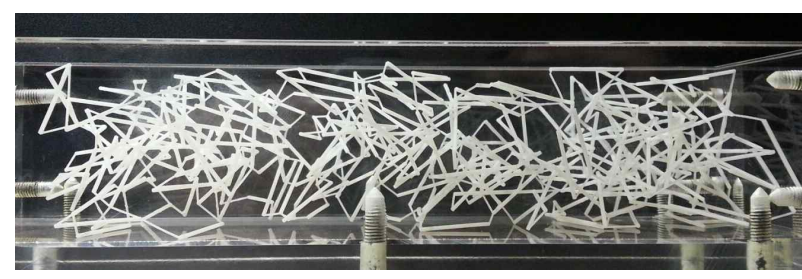

(b) Side view

Figure 11: Randomly-distributed printed fibers in the mold

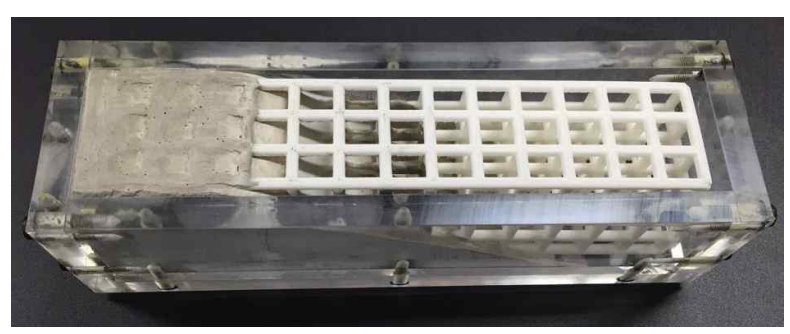

Figure 12: Aligned fiber filled with mortar (casting halted to show the reinforcement)

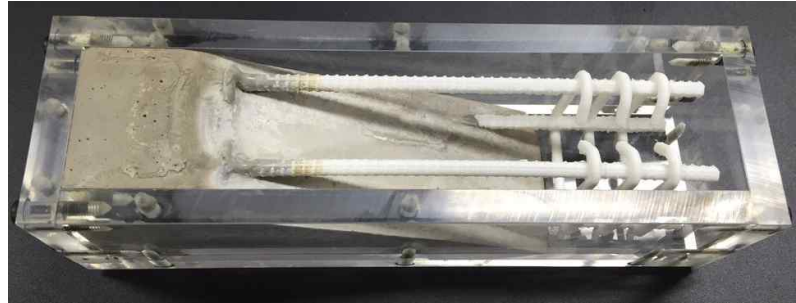

Figure 13: Printed rebar filled with mortar

\section{CONCLUSIONS}

The purpose of this research is to suggest 3D printing technology as a potential solution to common problems in the field of construction. Traditionally, when making fiber-reinforced composites, fibers were mixed with matrices at a variety of volume fractions. And Despite much research on how to control fiber distribution and how to detect the orientation of fibers using image technology, the procedure was still difficult.

With the adoption of $3 \mathrm{D}$ printing, controlling the coordinates, direction, and locations of the fibers is possible which leads to the accurate design of FRCCs. Although 3D printing techniques still have some challenges concerning modeling speed, printing material, and quality of printing, these difficulties might be overcome in few years with the development of $3 \mathrm{D}$ printing technique $[10,13]$. In this research, for economical reasons, photopolymer was used instead of materials such as metal, PVA, and others.

In this research, rebar, aligned fiber, and randomly distributed fiber were fabricated to examine the efficiency of 3D printing technique when making FRCCs. Even though the performance of connected fibers is not yet known, they are expected to improve the fracture behavior of cementitious composites. Therefore, further studies such as a three-point bending test with and without notches of the 3D printed fiber composite and other structures will be performed to verify the effectiveness of this research for the fracture behavior of FRCCs. 


\section{ACKNOWLEDGEMENTS}

This work is ongoing research supported by the EDISON (Education-research Integration through Simulation On the Net) Program through the National Research Foundation of Korea (NRF, funded by the Ministry of Science, ICT \& Future Planning (2014M3C1A6038855)

\section{REFERENCES}

[1] Corporation EPC. n.d. The History of Fibre Reinforcement. Retrieved from http://www. elastoplastic.com

[2] Kang, S.T., Bang, Y.L., and Koh, K.T. (2013). Prediction of fiber dispersion and orientation in fiber-reinforced cementitious composites. Journal of the Korea Concrete Institute 25, 39-42.

[3] Laranjeira, F., Grünewald, S., Walraven, J., Blom, C., Molins, C., and Aguado, A. (2011). Characterization of the orientation profile of steel fiber reinforced concrete. Materials and Structures 44(6), 1093-1111.

[4] Teßmann, M., Mohr, S., Gayetskyy, S., Haßler, U., Hanke, R., \& Greiner, G. (2010). Automatic determination of fiberlength distribution in composite material using 3D CT data. EURASIP Journal on Advances in Signal Processing, 2010(1), 1-9.

[5] Oh, J., Oh, J.S., and Jung, H.Y. (2014). Applicability to construction of 3D printing technology. Korean Society of Civil Engineers, 62, 38-44.

[6] Naaman, A.E., Alkhairi, F.M., and Hammoud, H. (1993). High Early Strength Fiber Reinforced Concrete (1st ed.). National Academy of Sciences, Washington.

[7] Naaman, A. E., and Najm, H. (1991). Bond-slip mechanisms of steel fibers in concrete. ACI Materials Journal, 88(2), 135-145

[8] Chua, C.K., and Leong, K.F. (2014). 3D Printing and Additive Manufacturing: Principles and Applications (4th ed.). World Scientific, Singapore.

[9] Chun, P., Lee, S.H., Cho, S.H., and Lim, Y.M. (2013). Experimental study on blast resistance of SIFCON. Journal of
Advanced Concrete Technology, 11, 144150.

[10]Wang, J. F., Zhang, L. Y., and Liu, P. (2015). 3D printing development study based on co-word analysis and multivariate statistics. Proceedings of the 2015 International Industrial Informatics and Computer Engineering Conference, 20812084.

[11] Wong, K. V., \& Hernandez, A. (2012). A review of additive manufacturing. ISRN Mechanical Engineering.

[12] Zhang, X., Le, X., Panotopoulou, A., Whiting, E., \& Wang, C. C. (2015). Perceptual models of preference in $3 \mathrm{~d}$ printing direction. ACM Transactions on Graphics (TOG), 34(6), 215.

[13] Scott. D. (2015 May 13). Metal Additive Manufacturing Market Continues Rapid Expansion. Retrieved from http://3d printing .com

[14]Lipson, H., \& Kurman, M. (2013). Fabricated: The New World of 3D Printing. John Wiley \& Sons. 